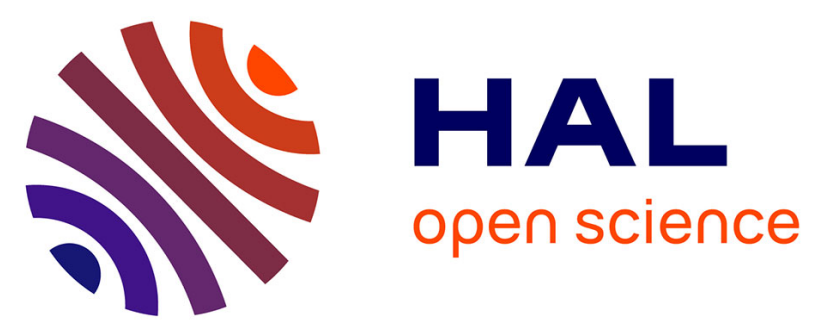

\title{
Complex Optical Index of HgTe Nanocrystal Infrared Thin Films and Its Use for Short Wave Infrared Photodiode Design
}

\author{
Prachi Rastogi, Audrey Chu, Tung Huu Dang, Yoann Prado, Charlie
} Gréboval, Junling Qu, Corentin Dabard, Adrien Khalili, Erwan Dandeu, Baptiste Fix, et al.

\section{To cite this version:}

Prachi Rastogi, Audrey Chu, Tung Huu Dang, Yoann Prado, Charlie Gréboval, et al.. Complex Optical Index of HgTe Nanocrystal Infrared Thin Films and Its Use for Short Wave Infrared Photodiode Design. Advanced Optical Materials, 2021, pp.2002066. 10.1002/adom.202002066 . hal-03161581

\author{
HAL Id: hal-03161581 \\ https://hal.science/hal-03161581
}

Submitted on 7 Mar 2021

HAL is a multi-disciplinary open access archive for the deposit and dissemination of scientific research documents, whether they are published or not. The documents may come from teaching and research institutions in France or abroad, or from public or private research centers.
L'archive ouverte pluridisciplinaire HAL, est destinée au dépôt et à la diffusion de documents scientifiques de niveau recherche, publiés ou non, émanant des établissements d'enseignement et de recherche français ou étrangers, des laboratoires publics ou privés. 


\section{Complex Optical Index of HgTe Nanocrystal Infrared Thin Films and its Use for Short Wave Infrared Photodiode Design}

Prachi Rastogi ${ }^{1 \$}$, Audrey Chu ${ }^{1,2 \$}$, Tung Dang ${ }^{1,3}$, Yoann Prado ${ }^{1}$, Charlie Gréboval ${ }^{1}$, Junling Qu ${ }^{1}$, Corentin Dabard ${ }^{1,4}$, Adrien Khalili ${ }^{1}$, Erwan Dandeu ${ }^{1}$, Baptiste Fix ${ }^{2}$, Xiang Zhen $\mathrm{Xu}^{2}$, Sandrine Ithurria $^{4}$, Gregory Vincent ${ }^{2}$, Bruno Gallas ${ }^{1}$, Emmanuel Lhuillier $^{{ }^{*}}$

1 Sorbonne Université, CNRS, Institut des NanoSciences de Paris, INSP, 75005 Paris, France.

${ }^{2}$ ONERA - The French Aerospace Lab, 6, chemin de la Vauve aux Granges, BP 80100, 91123 Palaiseau, France.

${ }^{3}$ Laboratoire de physique de l'Ecole Normale Supérieure, ENS, Université PSL, CNRS, Sorbonne Université, Université de Paris, 75005 Paris, France

${ }^{4}$ Laboratoire de Physique et d'Etude des Matériaux, ESPCI-Paris, PSL Research University, Sorbonne Université Univ Paris 06, CNRS UMR 8213, 10 rue Vauquelin, 75005 Paris, France.

Abstract: The limited investigations of the optical properties of HgTe nanocrystal (NC) thin films have reached a bottleneck for the electromagnetic design of devices. Using broadband ellipsometry, we determine the refractive index $(n)$ and the extinction coefficient $(k)$ for a series of $\mathrm{HgTe} \mathrm{NC}$ films relevant for infrared sensing applications. Electromagnetic simulations reveal that the $n$ value of $\mathrm{HgTe} \mathrm{NC}$ thin films can conveniently be approximated by its mean spectral value $n=2.35 \pm 0.15$. We then use this complex optical index to design a diode with $(i)$ a reduced amount of $\mathrm{Hg}$ containing material (thin film $<150 \mathrm{~nm}$ ) and (ii) a thickness of the device better-matched with the carrier diffusion length. We demonstrate that introducing an aluminum grating onto the transparent conductive electrode leads to an enhanced absorption while reinforcing the work-function difference between the two electrodes. Broadband $(\approx 1 \mu \mathrm{m})$, non-polarized and strong absorption up to $100 \%$

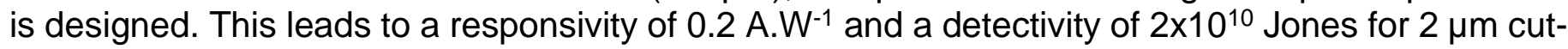
off wavelength at room-temperature, while the time response is as short as $110 \mathrm{~ns}$.

\$ these two authors have equal contributions

To whom correspondence should be sent: el@insp.upmc.fr 


\section{Introduction}

Infrared nanocrystals (IR NCs) raise great promises for the design of low-cost IR sensors. ${ }^{1,2}$ This especially includes the demonstration of focal plane arrays ${ }^{3}$ in which the active layer is made of NCs operating in the short-wave, ${ }^{4}$ extended short-wave ${ }^{5,6}$, and mid-wave IR. ${ }^{7,8}$ Compared with epitaxially-grown semiconductors, colloidal NCs simplify the coupling to the read-out circuit ${ }^{9-11}$ (i.e. no hybridization step through indium bumps). Beyond the cost reduction, it also eases pixel pitch reduction, bringing it closer to the diffraction limit, ${ }^{12,13}$ from 15 to $5 \mu \mathrm{m}$ typically.

Among potential material candidates, $\mathrm{HgTe}$ offers the wider spectral tunability. It can be grown under highly confined forms such as nanoplatelets ${ }^{14-16}$ with band-edge around $800 \mathrm{~nm}$ as well as at larger sizes (>> Bohr radius) with NCs absorbing in the $\mathrm{THz}$ range. ${ }^{17,18}$ Over the recent years, significant progresses have been made on the device performance as their geometries have been improved. Compared to initial poor ligand-exchanged films deposited on interdigitated electrodes, complex photodiodes 4,19 and phototransistors presenting a reduced dark current and an enhanced photocarrier dissociation are now proposed. The control of the light-matter coupling is certainly one of the directions that have led to the most recent improvements. Cavities ${ }^{20,21}$ and plasmonic resonators ${ }^{22-24}$ have been introduced to obtain strongly-absorbing thin films. The interest for the light-matter coupling control is not limited to the material absorption. It also raises interest in light emission ${ }^{25}$ and also potentially in lasing regarding the recent demonstrations of low threshold stimulated emission in $\mathrm{HgTe}$ NCs. ${ }^{26}$

However, the design of such optically active nanostructures relies on the accurate understanding of the electromagnetic properties of the involved materials. Indeed, the design of such cavities and resonators relies on electromagnetic simulations based on Finite-Difference Time-Domain (FDTD) or Rigorous Coupled-Wave Analysis (RCWA) methods. These simulations use the complex optical indices of the material as an input. While systematic measurements of the dielectric constant have been conducted for cadmium and lead chalcogenides, ${ }^{27}$ no studies have been carried out on $\mathrm{HgTe}$, preventing accurate electromagnetic designs. Current strategies consider that the complex part of the optical index and the absorption spectrum can be assimilated, its value at the band edge is calibrated using the absorption coefficient. ${ }^{28}$ The real part is often taken as a constant value around 2 , which is smaller than the bulk refractive index $\left(n_{\mathrm{HgTe}}^{\text {bulk }} \in[3-4]\right)^{29-32}$ due to the presence of organics and voids. Even though this value $n_{H g T e}^{N C}=2$ leads to realistic simulations ${ }^{20}$, it lacks of experimental support. The bulk complex optical index also remains poorly determined due to a lack of consistency between data with a broad range of material contents (from $\mathrm{HgCdTe}$ with various alloy contents ${ }^{33,34}$ ) and spectral ranges (from visible to far IR). ${ }^{35,36}$ This suggests that additional experimental data are required to be further used as input for the electromagnetic designs of IR NCbased device structures.

In this paper, we first determine the complex optical index of $\mathrm{HgTe} \mathrm{NC}$ thin films using broadband (350 - $2500 \mathrm{~nm}$ ) ellipsometry measurements for a series of NCs relevant for short-wave and midwave IR detection. We then focus on the importance of designing efficient light resonators to enhance the absorption of thin films, to reduce $\mathrm{Hg}$-based materials consumption and to enhance charge transport efficiency. Thus, instead of focusing on thick structures as it has been done by other groups, we target thin devices $(<150 \mathrm{~nm})$. We show that the introduction of an aluminum grating enables broadband and high absorption ( $75 \%$ mean absorption over $\approx 1 \mu \mathrm{m}$ ), leading to a high detectivity $>10^{10}$ Jones at room-temperature while presenting fast operation (110 ns as decay time). 


\section{Results and discussion}

\section{Revealing the complex optical index of $\mathrm{HgTe} \mathrm{NC}$ thin films}

We start by growing a series of HgTe NCs (see microscopy image in Figure 1a) with various sizes corresponding to band-edge energies from $1.05 \mathrm{eV}\left(1.2 \mu \mathrm{m}, 8500 \mathrm{~cm}^{-1}\right)$ down to $0.25 \mathrm{eV}(5 \mu \mathrm{m}$, $2000 \mathrm{~cm}^{-1}$ ), see Table 1 and Figure $1 \mathrm{~b}$. We use the Keuleyan's procedure ${ }^{37}$ where mercury chloride reacts with trioctylphosphine:tellurium in oleylamine. The temperature of the reaction is used to tune the particle size. In the following text, each population is labeled $\mathrm{HgTe} N \mu$, where $N$ is the wavelength at the band-edge energy in $\mu \mathrm{m}$.

The solutions obtained are deposited into thin films (see inset of Figure 1a). We use a liquid phase ligand-exchange leading to an ink (NCs capped with short ligands in solution: $\mathrm{HgCl}_{2}$ combined with mercaptoethanol) later deposited as a thin film using spin-coating. Complementarily, we also prepare films using another surface chemistry (ethanedithiol: EDT), through a solid-state ligandexchange approach (see methods) to reveal the effects of the ligands on the complex optical index.
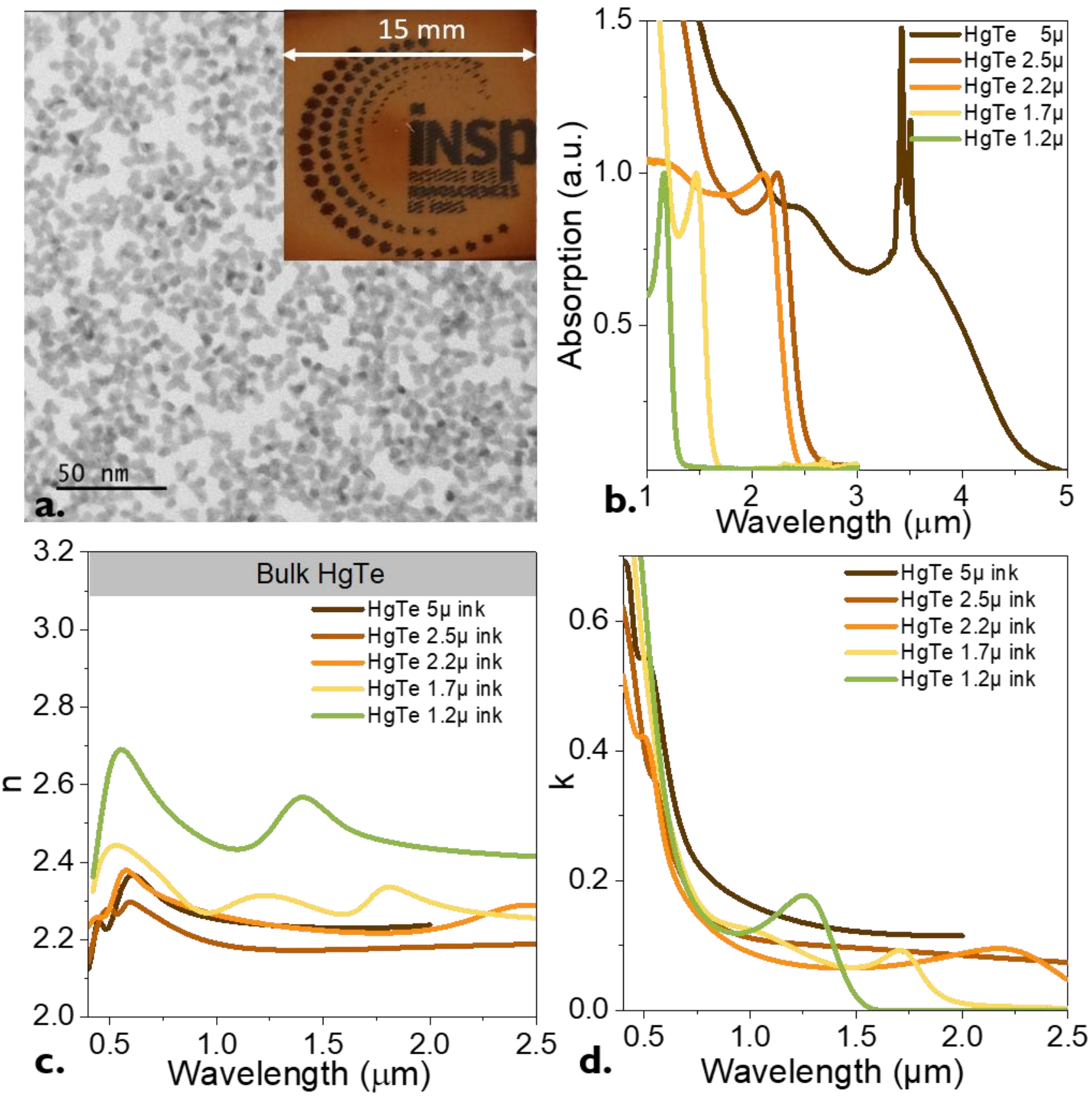
Figure 1 a. Transmission electron microscopy image of HgTe NCs with band-edge energy around $0.5 \mathrm{eV}(\mathrm{HgTe} 2.5 \mu)$. The inset is a picture of the film. b. Absorption spectra for various sizes of $\mathrm{HgTe}$ NCs. C. Refractive index as a function of the wavelength for various sizes of HgTe NCs. d. Extinction coefficient as a function of the wavelength for various sizes of HgTe NCs.

We measure then the optical index for each film using a broadband spectroscopic ellipsometer (350 $\mathrm{nm}-2500 \mathrm{~nm}$ ), see Figure S2-S11. It enables spectrally resolved determination of the real and imaginary parts of the optical index (and so the refractive index, $\mathrm{n}$ and the extinction coefficient, $\mathrm{k}$ ) up to the band-edge for three populations of $\mathrm{HgTe} \mathrm{NC}$ thin films ( $\mathrm{HgTe} 1.2 \mu, 1.7 \mu$ and $2.2 \mu)$, see Figure $1 \mathrm{c}-\mathrm{d}$. We also provide the data associated with these spectra in the supporting information. Repeating the measurement on a series of films made of $\mathrm{HgTe} 1.7 \mu$ (Figure S9-10) with various thicknesses, we establish the error bar on the magnitude of the refractive index and extinction coefficient to be respectively 0.1 and 0.02 .

Table 1 Optical properties for HgTe NC films. * indicates that the band-edge value has been obtained from transmission measurements. The absorption coefficient ( $\alpha$ ) is defined so that the absorbance $A$ is equal to $A=\alpha L$ with $L$ the film thickness. $\alpha$ can be related to $k$ using the expression $\alpha=\frac{4 \pi k}{\lambda \cdot \ln (10)}$ with $\lambda$ the wavelength.

\begin{tabular}{cccccc}
\hline Sample & $\begin{array}{c}\text { Exciton peak } \\
\text { wavelength } \\
(\boldsymbol{\mu m})\end{array}$ & $\begin{array}{c}\text { Exciton peak } \\
\text { energy }(\mathbf{e V})\end{array}$ & $\begin{array}{c}\text { Abs coeff. at } \\
\text { the band-edge } \\
\left(\times 10^{3} \mathbf{c m}^{-1}\right)\end{array}$ & $\begin{array}{c}\text { Mean } \\
\text { value of } \mathbf{n} \\
\mathbf{n}_{\text {eq }}\end{array}$ & kex \\
\hline HgTe 1.2 $\boldsymbol{\mu}$-ink & 1.2 & 1.05 & 8.2 & 2.5 & 0.18 \\
\hline HgTe 1.7 $\mu$-ink & 1.7 & 0.74 & 4.2 & 2.4 & 0.13 \\
\hline HgTe $2.2 \mu$-ink & 2.2 & 0.57 & 2.6 & 2.25 & 0.1 \\
\hline HgTe $2.5 \mu$-ink & 2.5 & 0.50 & 2.6 & 2.2 & $0.12^{*}$ \\
\hline HgTe $5 \mu$-ink & 5 & 0.25 & 1.8 & 2.25 & $0.13^{*}$ \\
\hline \hline
\end{tabular}

In the extinction coefficient spectra, see Figure 1d, the spectral shapes expected from absorption measurements is recovered (Figure 1b). The refractive index reveals more unexpected results. First, typical values obtained for $\mathrm{n}$ are below the bulk value $\left(n_{\mathrm{HgTe}}^{\text {bulk }} \in[3-4]\right)^{29-32}$, grayed area in Figure 1c). These results were anticipated due to the possible presence of organics and voids within the NC array. However, these values seem to be weakly connected to the band-edge energy or to the particle size. Regardless of the particle sizes or the surface chemistries, all samples fit with $n=2.35$ \pm 0.15 . This value is larger than the one conveniently used in the electromagnetic design $(n=2)^{22,24}$ but matches well with the value recently used by Tang and coworkers ${ }^{38}$. This encourages a clear update of the input parameters for future simulation works. It is also worth noticing that the surface chemistry/film deposition method (see Figure S5, 7 and 8) plays a larger role in the final $n$ value than the particle size as a change in the film preparation can reduce the refractive index by 0.2 (figure S8). Finally, consistent with the Kramers-Kronig relations, $n$ has a relatively weak spectral dependence, with variations around 0.1 (i.e. $\approx 5 \%$ ) over the whole spectral range, while the $k$ values increase by a factor 7 over the same range. This raises the question of whether or not, we should include the spectral dependence of $n$ in future simulations.

To answer this question, we build and optically characterize an HgTe NC-based diode structure previously reported in the literature. ${ }^{19}$ This diode is designed for extended short-wave IR detection 
and relies on the glass/FTO/HgTe2. $2 \mu / \mathrm{HgTe} 1.7 \mu /$ gold stack, see a scheme of the structure in Figure 2a. The FTO (fluorine-doped tin oxide) is used as an electron collecting layer and the $\mathrm{HgTe} 2.2 \mu$ $(0.55 \mathrm{eV}, 2.2 \mu \mathrm{m})$ is the absorbing ambipolar layer. The $\mathrm{HgTe} 1.7 \mu$ is used as a unipolar barrier and the gold as the hole extractor. ${ }^{19,39}$

We then simulate the absorption spectra of the above complex stack structure using $n$ values obtained from the spectrally resolved measurements (from Figure $2 \mathrm{c}$ ) and constant $n$ values, see Figure $2 \mathrm{~b}$. We observe that the simulated curve including the spectral dependence of $n$ clearly matches the one corresponding to $n=2.25$. This value appears to be the mean value obtained as we average $n$ over the whole spectral range, see Table 1. In other words, it appears that neglecting the spectral dependence of the refractive index is a good approximation, and only the updated average value needs to be considered in future simulations. To further confirm this, we compare the experimental spectrum (measured from reflectance) with the simulated one from the stack and obtain a very reasonable agreement, as shown in Figure $2 \mathrm{c}$.
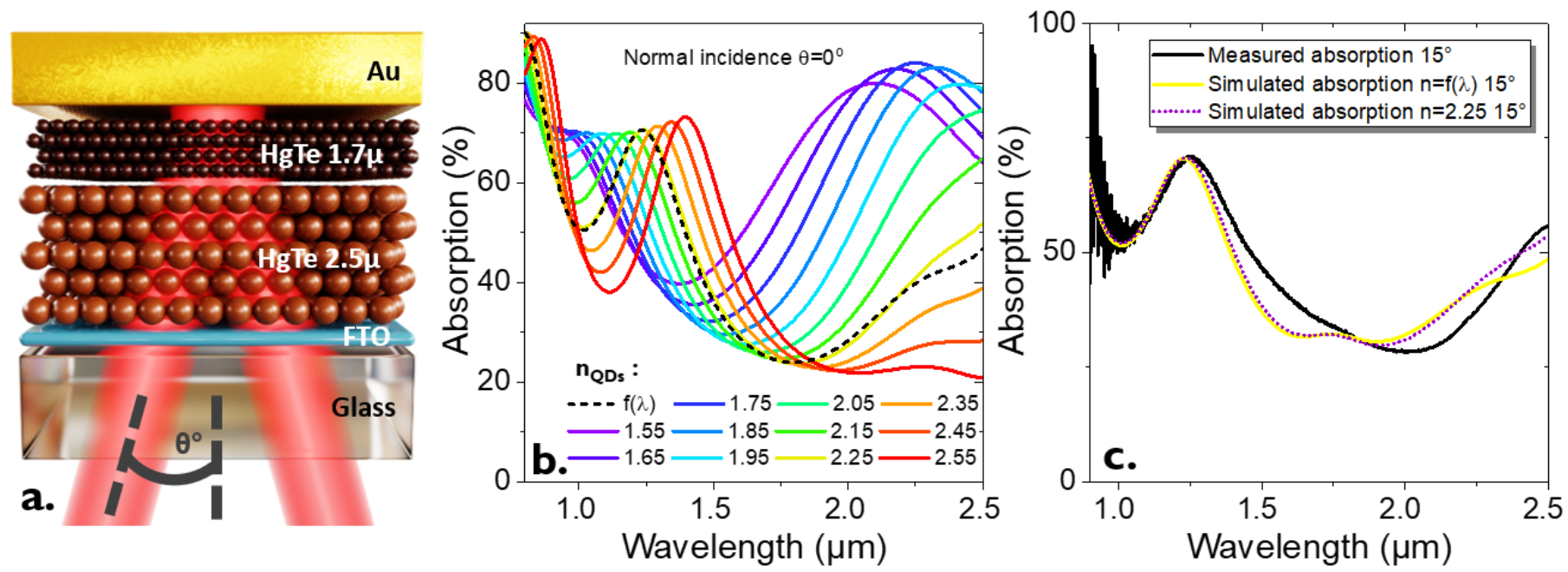

Figure 2 a. Scheme of a HgTe NC-based diode dedicated to infrared photodetection in the extended short-wave infrared. $b$. Simulated absorption spectra for the device of part a, while the refractive index of the HgTe 2.2 $\mu$ layer is spectrally resolved (dashed line) and while different constant values of $n$ are used. c. Measured and simulated spectra for the structure described in part $a$.

\section{Design of a short-wave infrared diode}

Following this first study, we use the measured complex optical indices to design a diode operating in the short-wave IR around telecom wavelengths $(\approx 1.55 \mu \mathrm{m})$. Ackerman et al. observed that a layer of $\mathrm{Ag}_{2} \mathrm{Te} \mathrm{NCs}$ (see figure S1), treated using $\mathrm{HgCl}_{2}$ (which induces a cation exchange), behaves as a high-performing hole extractor for a HgTe NC thin film. ${ }^{40}$ They demonstrated this from $2.2 \mu \mathrm{m}^{40}$ and up to $5 \mu \mathrm{m},{ }^{41}$ but did not mention any shorter wavelengths. Inspired by their concept, we build a diode based on the following stack glass/FTO/HgTe1.7 $/ \mathrm{Hg}: \mathrm{Ag}_{2} \mathrm{Te} / \mathrm{gold}$, see Figure $3 \mathrm{a}$. Under illumination, the I-V characteristic of such a diode presents a clear open-circuit voltage (Figure $3 \mathrm{~b}$ ) while having a fast time response (few $\mu \mathrm{s}$ - setup limited), see Figure 3c.

We simulate the absorption (taken as $1-R$, with $R$ the reflectivity, since the transmission is null in the diode geometry due to the presence of a metallic reflective film used as an electrode) of this diode for different film thicknesses, see Figure $3 \mathrm{~d}$. We assume in this simulation that the $\mathrm{Hg}: \mathrm{Ag}_{2} \mathrm{Te}$ layer has a similar optical index as the underneath $\mathrm{HgTe}$ film. In this absorption map, yellow lines depending on the thickness correspond to Fabry-Perot cavities, see Figure $3 \mathrm{~d}$. Around $1.7 \mu \mathrm{m}$ the absorption is a bit modulated due to the exciton peak of HgTe. The cavities are formed thanks to the low refractive index of the substrate $(n=1.5)$ compared to the NC film and the presence of the 
gold mirror on the other side of the diode. Absorption up to $70 \%$ can then be obtained for device as thin as $150 \mathrm{~nm}$. However, in order to get effective performance, the absorption needs to occur within the NC layer. If the absorption is located within the contacts, no photocurrent is generated and only thermal losses are observed. Thus, we refine the map by considering the absorption only within the $\mathrm{NCs}$, see Figure 3e. It appears that the first resonance is mainly induced by losses in the contacts. To achieve absorption beyond $50 \%$ in the NCs at $1.55 \mu \mathrm{m}$, a thickness between 400 and $500 \mathrm{~nm}$ range for the NC film is required. We fabricate a series of device in which the thickness of the absorbing layer is then increased. From an optical point of view, the thicker the absorbing layer is, the higher the absorption will be. Experimentally, we observe an increase of the responsivity and the open-circuit voltage with the increase of device thickness, see Figure $3 f$. However, for thicknesses above $300 \mathrm{~nm}$, we observe a decrease of the photoresponse, a loss of the rectifying behavior, and a drastic reduction of the success ratio to build the device without electrical shorts (Figure 3f). This drop of the performance highlights ( $I$ ) the difficulty to build thick qualitative films and (ii) charge collection becomes less efficient when transport occurs over distances longer than the diffusion length, which has been estimated around $170 \mathrm{~nm}$ from a previous study. ${ }^{42}$
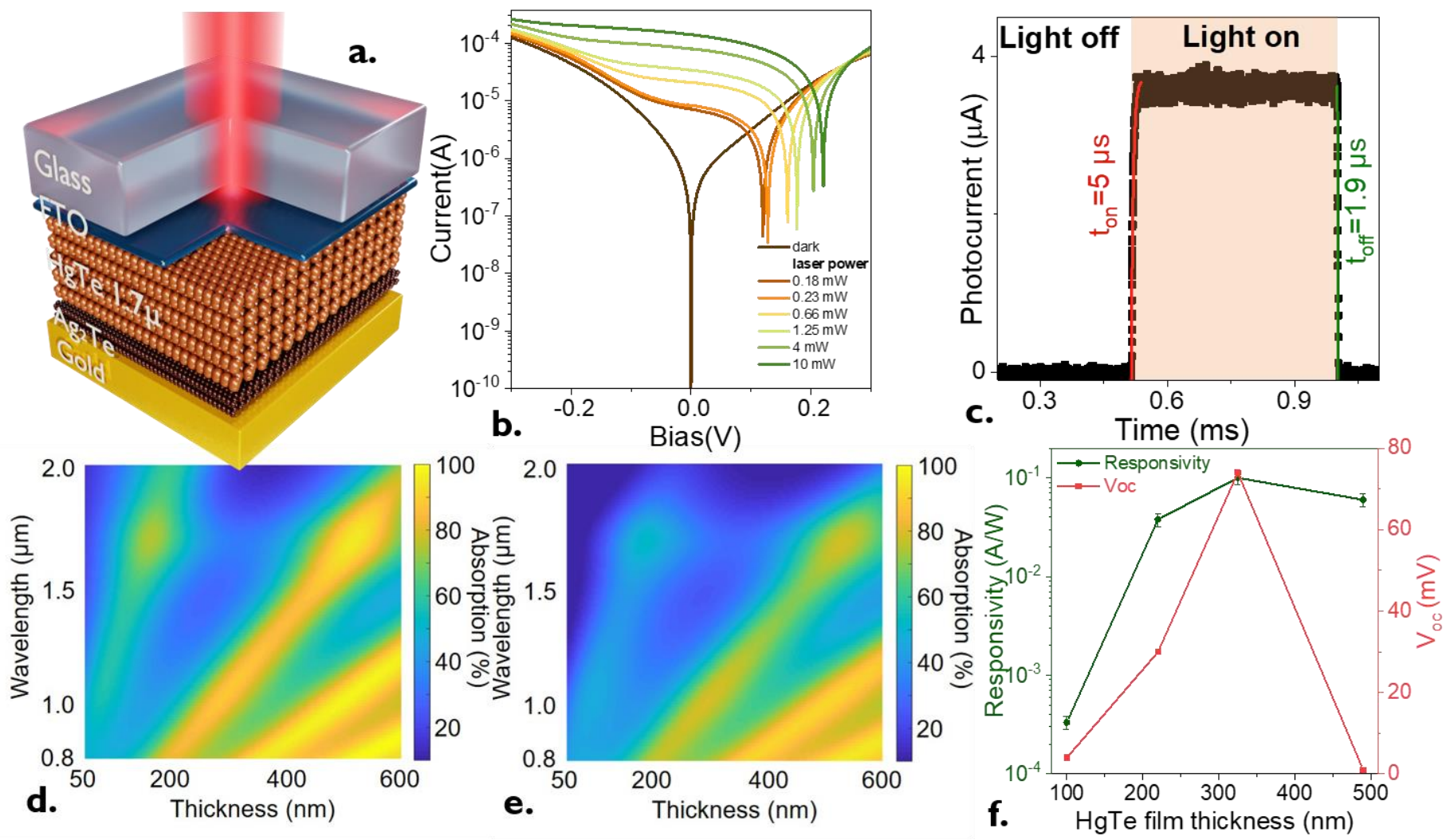

Figure 3 a. Scheme of the diode based on glass/FTO/HgTe1.7 $\mu / \mathrm{Hg}: \mathrm{Ag}_{2} \mathrm{Te} / \mathrm{Gold}$ stack. b. IV curves in the dark and under illumination for the diode depicted in part a. c. Time response of this diode under a pulse of light. $d$. Simulated total (contact $+N C s$ ) absorption spectra of the diode depicted in part a while the thickness of the absorbing layer $(\mathrm{HgTe} 1.7 \mu)$ is tuned. e. Simulated NC absorption (i.e. excluding contact) spectra of the diode depicted in part a while the thickness of the absorbing layer ( $\mathrm{HgTe} 1.7 \mu$ ) is tuned. $f$. Responsivity and open-circuit voltage of the diode depicted in part a while the thickness of the absorbing layer (HgTe 1.7 $\mu$ ) is tuned. The error bar corresponds to a 15\% error relative to the fluctuation of incident light power resulting from optical misalignment.

\section{Need for resonators}

To solve this issue, we aim to tune the light-matter interaction by coupling the absorbing layer with cavities and resonators. The first goal of this resonator is to concentrate the electromagnetic field into a slab of NC thinner than the absorption depth (few $\mu \mathrm{m}$ ) in order to absorb most of the light on 
a device that has a thickness compatible with the transport diffusion length. The second motivation is to obtain a certain spectral shape just by the design of the resonator. In the case of NC-based devices, this concept remains poorly explored while, for the third-generation solar cells, the coupling of a thin absorbing layer with resonators already enables broadband absorption by combining several types of resonances. Up to 9 resonances have been associated to cover the whole visible range. ${ }^{43}$ Tang et al. have proposed to stack a Fabry-Perot cavity made of a $\mathrm{SiO}_{2}$ spacer with a metal-insulator-metal resonator to enhance absorption. ${ }^{20}$ Their structure leads to impressive responsivity but still relies on a thick absorbing film and requires multiple fabrication steps which might be incompatible with the targeted low-cost of NC-based devices. Here, we demonstrate that electromagnetic design can simplify this structure while obtaining up to $100 \%$ absorption of the incident light.

Our idea is to use the same electrically efficient structure (glass/FTO/HgTe1.7 $\mu / \mathrm{Hg}_{\mathrm{Ag}} \mathrm{ATe} / \mathrm{Gold}$ ) as before, but functionalizing it with a grating. A 1D grating can be used to achieve polarized absorption (see Figure 4a) or a 2D grating (see Figure 5a) to maximize the absorption.

\section{3a. 1D resonator with polarized response}

To design a guided mode resonator (GMR), we functionalize the diode with a metallic grating. The typical period $p$ of the grating is $\lambda / \mathrm{n}$. For resonance at $1.55 \mu \mathrm{m}$, this leads to $p \approx 700 \mathrm{~nm}$ requiring an additional e-beam lithography step to be fabricated. To avoid possible damages of the active layer due to baking or exposure to bad solvent, we choose to build the grating on the FTO side (i.e. before NC deposition). This strategy minimizes the degradation of the NCs. In addition, the grating should not affect the electrical operation of the diode. FTO being the electron extractor, we choose to use a low work-function metal to fabricate the resonator. We observe that silver deposited onto $\mathrm{HgTe}$ $\mathrm{NC}$ film quickly turns to a black (i.e. a non-reflective layer), likely due to the silver telluride formation through a cation exchange process. We consequently chose to use aluminum to design the metallic grating. A sketch of the device is shown in Figure 4a.

The presence of the grating now enables to achieve strong absorption ( $>90 \%$ for the Transverse Magnetic (TM) polarized light and $60 \%$ for non-polarized light) for a device as thin as $\approx 150 \mathrm{~nm}$, see Figure $4 \mathrm{~d}$-e. The absorption spectrum reveals the presence of two contributions. At $1.55 \mu \mathrm{m}$, a first resonance along the TM polarization (see Figure 4d) results from a GMR, see Figure 4f. The absorption map in this polarization (Figure $4 b$ ) confirms that the absorption mostly (70\%) occurs within the semiconductor.

At $1.27 \mu \mathrm{m}$, we observe a second resonance. The dispersion map along the Transverse Electric (TE) polarization (Figure $4 \mathrm{~g}$ ) shows that this resonance is barely dispersive (i.e. no $k_{x}$ dependence) revealing its Fabry-Perot nature. This mode is similar to the one observed in the structure without grating (the cavity corresponds to the NC layer between gold and FTO) and consequently only $40 \%$ of the absorption occurs in the NCs for such thin structure, see Figure 4c. 

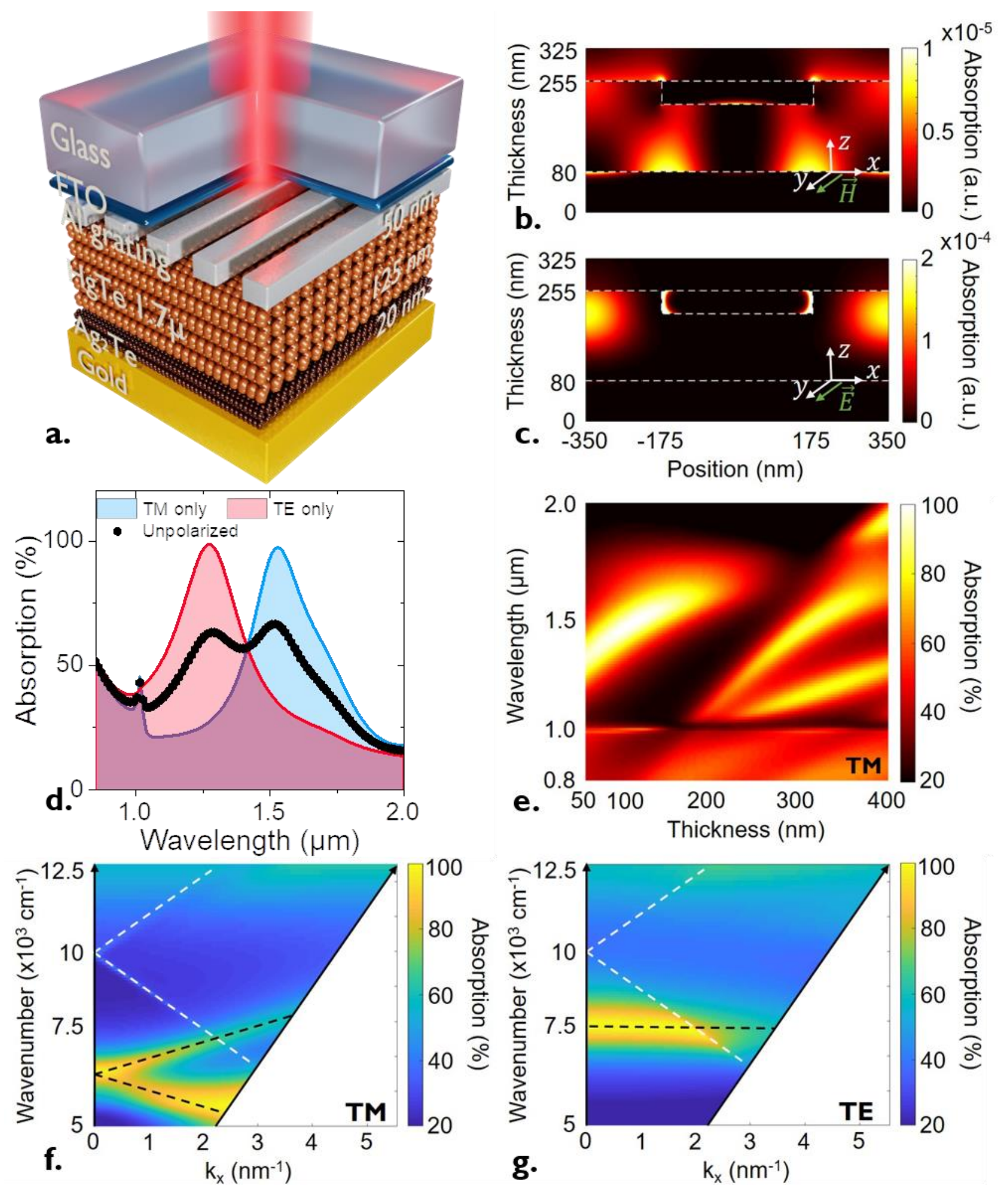

Figure 4 a. Sketch of a the diode based on glass/FTO:Al-1D grating/HgTe1.7 $\mu / \mathrm{Hg}: \mathrm{Ag}_{2} \mathrm{Te} / \mathrm{Gold}$ stack. b. (resp c.) Simulated absorption map along with the TM (resp TE) polarization at the maximum of the absorption for this polarization. $d$. Simulated absorption spectra for a 125-nm thick layer of $\mathrm{HgTe}$ $1.7 \mu$ for TM, TE polarized and unpolarized incident light. e. Simulated absorption map as a function of the wavelength and the thickness of the $\mathrm{HgTe} 1.7 \mu$ layer along with the TM polarization. $f$. (resp g.) Angle-resolved specular absorption simulation as a function of $\sigma=1 / \lambda$, the wavenumber, and $k_{x}=2 \pi \cdot \sin (\theta) / \lambda$ for the TM mode (resp TE). Black dashed lines correspond to the absorption peak. White dashed lines correspond to the appearance/disappearance limit of the first diffracted order in the substrate. 


\section{3b. 2D resonator with strong non-polarized absorption}

To further maximize the absorption, a non-polarized structure is designed. In this device the metallic stripes are replaced by metallic dots, while the period remains unchanged, see a sketch of the device in Figure 5a. By doing so, the TE and TM modes of the previous structure become degenerated under normal incidence, see Figure 5f-g. The absorption spectrum looks similar to the 1D case but the TE and TM nature are now entangled for each peak. As a result, it becomes possible to achieve a $100 \%$ absorption (Figure $5 d-e$ ) even for non-polarized light, while having a broadband absorption which occurs mostly within the NCs $(62 \%$ in the NC for the resonance at $1.26 \mu \mathrm{m}$ and $64 \%$ for the one at $1.55 \mu \mathrm{m}$ ), see the absorption map in Figure $5 \mathrm{~b}$-c.
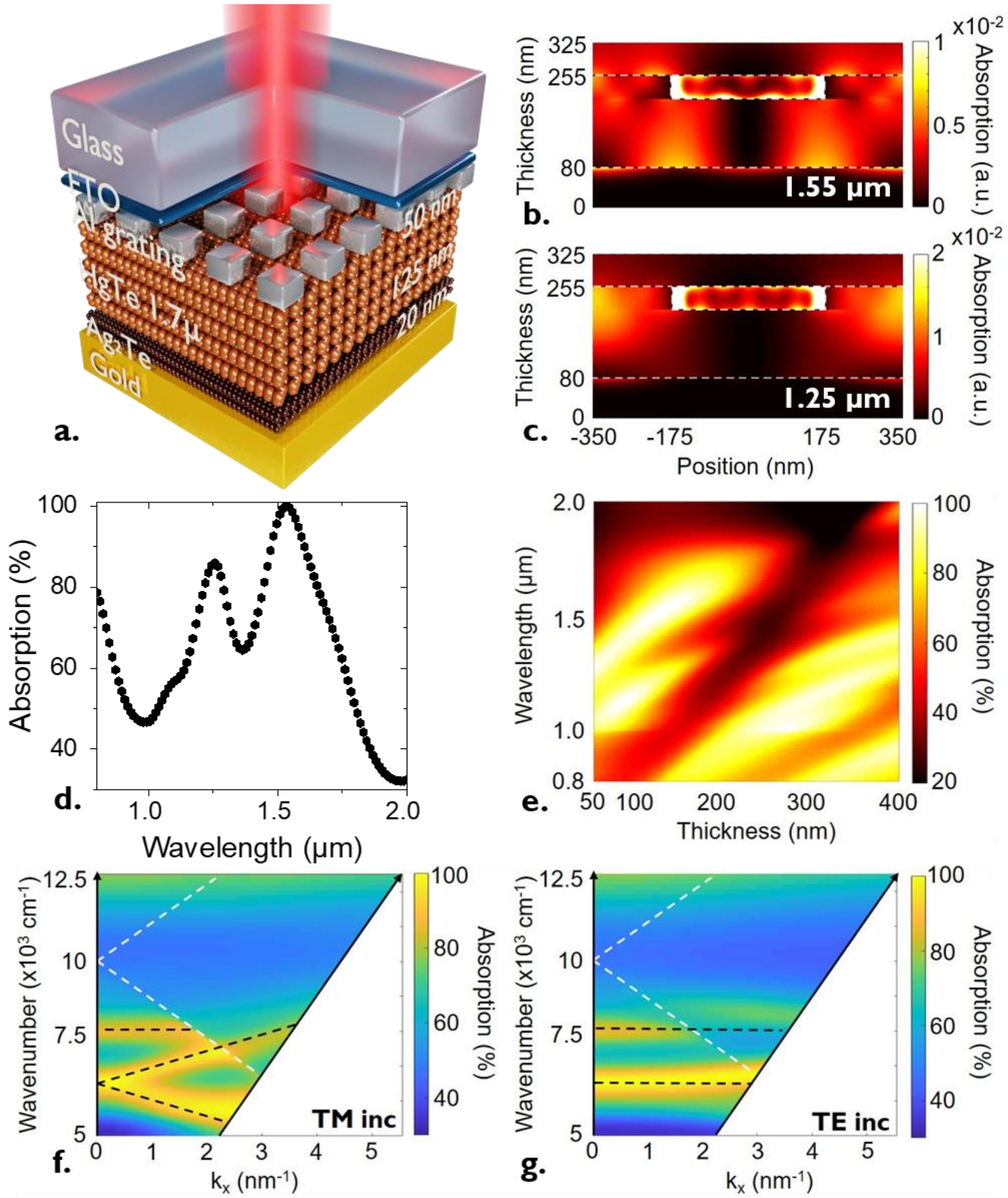

Figure $5 \mathrm{a}$. Sketch of a the diode based on glass/FTO:Al-2D grating/HgTe1.7 $\mu / \mathrm{Hg}: \mathrm{Ag}_{2} \mathrm{Te} / \mathrm{Gold}$ stack. b. (resp c.) Simulated absorption map along with the TM (resp TE) polarization at $1.55 \mu \mathrm{m}$ (resp 
$1.25 \mu \mathrm{m}) \mathrm{d}$. Simulated absorption spectrum for a $125 \mathrm{~nm}$ thick layer of $\mathrm{HgTe} 1.7 \mu$ for an unpolarized incident light. e. Simulated absorption map as a function of the wavelength and the thickness of the $\mathrm{HgTe} 1.7 \mu$ layer along the TM polarization. $f$. (resp g.) Angle-resolved specular absorption simulation as a function of $\sigma=1 / \lambda$, the wavenumber, and $k_{x}=2 \pi \cdot \sin (\theta) / \lambda$ for the TM mode (resp TE). Black dashed lines correspond to the absorption peak. White dashed lines correspond to the appearance/disappearance limit of the first diffracted order in the substrate.

\section{Short-wave infrared photodiode with broadband response based on $<150 \mathrm{~nm} \mathrm{NC}$ film}

We then fabricate the diode designed in the previous part. Compared to the diode without the grating, the fabrication requires an additional step of e-beam lithography (figure S13) but the benefit of the grating is clearly observed (Figure 6a, S14-16). Not only the magnitude of the response is increased, but also the increase of the photocurrent occurs over a broad range $(>1 \mu \mathrm{m})$. This spectral shape is enabled by the combination of the nanocrystal band edge with the two resonances induced by the grating. Those two resonances are slightly blue-shifted compared to the targeted design (Figure $5 \mathrm{~d}$ ) indicating that the final film thickness is thinner than initially targeted (135 nm instead of 150 $\mathrm{nm}$ ). This thickness fluctuation in the film is currently the physical factor limiting the exact reproducibility of the performances from device to device, see Figure S15-16.

The enhancement of the spectrally integrated photoresponse $(x 4.3)$ is also larger than the one expected from optical simulations (x 2 or an increase from $40 \%$ without grating to $80 \%$ of absorption in the presence of the grating according to simulations). To understand the origin of this additional enhancement, we also fabricate a diode with a gold (instead of Al) grating. In this case, a photoresponse is still observed (Figure 6d), but the IV curves are no longer rectifying (figure S14) and the open-circuit voltage under illumination drops to almost zero, see Figure 6c. This confirms that the presence of metallic nanostructures onto the FTO is not only acting as an optical grating but also strongly affects the work-function of the electron extracting electrode. If gold is added on the FTO, the asymmetry of the diode gets weaker, and the Voc is reduced, while, as a low workfunction metal, the aluminum is further enhancing the work-function difference with the top gold electrode, leading to an increased Voc. Thus, the aluminum used to design the grating is also involved in the electrical operation of the diode.

Under broadband blackbody illumination $\left(\mathrm{T}_{\mathrm{BB}}=980^{\circ} \mathrm{C}\right.$ combined with $\mathrm{Ge}$ filter to suppress light below $1.8 \mu \mathrm{m}$ ), taking advantage of the two optical resonances, we observe a response of $0.2{\mathrm{~A} . \mathrm{W}^{-1}}^{-1}$ $(E Q E=14 \%)$ at room temperature operation and with a cut-off wavelength of $2 \mu \mathrm{m}$. This value is close to the performance obtained by Ackerman et al. ${ }^{40}$ for an $\mathrm{HgTe}$ diode at slightly longer cut-off wavelengths $(2.2$ and $2.3 \mu \mathrm{m})$, while their diode was relying on a three-times thicker $(400 \mathrm{~nm})$ film. A comparison of this diode performance with state-of-the-art HgTe NC-based IR sensors is provided in Table 2. The noise of this structure is $1 / f$ limited. The best performing device leads to a specific detectivity of $2 \times 10^{10}$ Jones for $0 \mathrm{~V}$ operation at room-temperature and at $1 \mathrm{kHz}$ signal. Under a short pulse ( $<2 \mathrm{~ns}$ ) of light illumination, we found a time response of $110 \mathrm{~ns}$ for the device, see Figure 6e. 

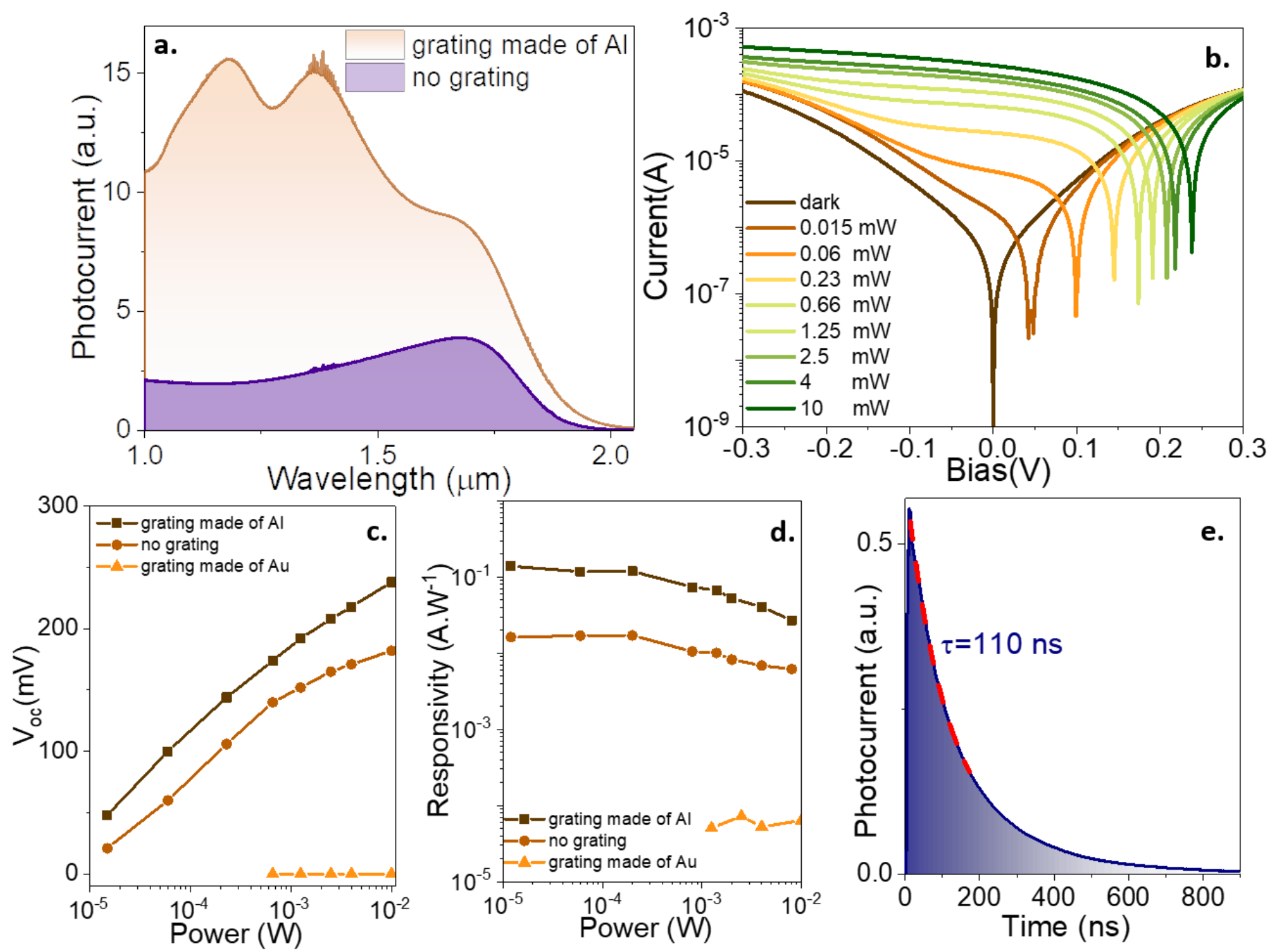

Figure 6 a. Photocurrent spectra for the glass/FTO./HgTe1.7 $/ \mathrm{Hg}: \mathrm{Ag}_{2} \mathrm{Te} / \mathrm{Gold}$ stack with and without GMR Al grating. b. IV curves for the glass/FTO:Al-2D grating/HgTe1.7 $/ \mathrm{Hg}: \mathrm{Ag}_{2} \mathrm{Te} / \mathrm{Gold}$ stack under different incident power. The illumination is performed with a $1.55 \mu \mathrm{m}$ laser diode. $c$. Open circuit voltage of the diode as a function of the incident power without GMR grating and with an Au or Al based grating. Also see Figure $S 11$ for associated IV curves. $d$. The responsivity of the glass/FTO:Al-2D grating/HgTe1.7 $/ \mathrm{Hg}: \mathrm{Ag}_{2} \mathrm{Te} / \mathrm{Gold}$ stack as a function of the incident power using a $1.55 \mu \mathrm{m}$ laser as source. e. Current as a function of time while the sample has been illuminated by a short (<2 ns) pulse of light (355 nm). Measurements are conducted under a $0 \mathrm{~V}$ bias operation and in ambient conditions.

Table 2: Comparison of state-of-the-art HgTe NC-based IR sensors. PC stands for a photoconductive device, while $P V$ relates to a photovoltaic operation and PT to a phototransistor mode.

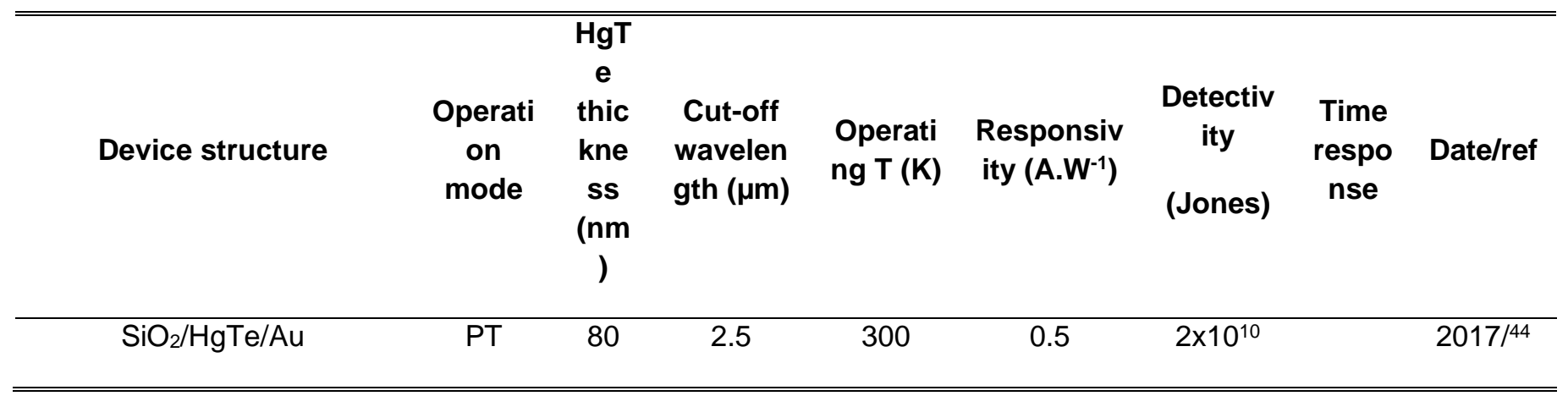




\begin{tabular}{|c|c|c|c|c|c|c|c|c|}
\hline $\begin{array}{c}\mathrm{Al}_{2} \mathrm{O}_{3} / \mathrm{ITO} / \mathrm{HgTe}: \mathrm{EDT} / \mathrm{Ag}_{2} \mathrm{~T} \\
\mathrm{e} / \mathrm{Au}\end{array}$ & PV & 400 & 5 & 230 & 0.1 & $10^{9}$ & $1 \mu \mathrm{s}$ & $2018 / 41$ \\
\hline $\begin{array}{c}\mathrm{Al}_{2} \mathrm{O}_{3} / \mathrm{ITO} / \mathrm{Au}- \\
\mathrm{disc} / \mathrm{HgTe} / \mathrm{Au} / \mathrm{ITO} / \mathrm{SiO}_{2} / \mathrm{Au}\end{array}$ & PV & 400 & 4.5 & 85 & 1.62 & $4 \times 10^{11}$ & - & $2018 / 20$ \\
\hline $\begin{array}{c}\mathrm{FTO} / \mathrm{TiO}_{2} / \mathrm{HgTe}: \mathrm{EDT} / \mathrm{MoO}_{3} / \\
\mathrm{Au}\end{array}$ & PV & 200 & 2.5 & 300 & 0.001 & $3 \times 10^{8}$ & - & $2018 / 19$ \\
\hline $\begin{array}{c}\mathrm{Al}_{2} \mathrm{O}_{3} / \mathrm{ITO} / \mathrm{Bi}_{2} \mathrm{Se}_{3} / \mathrm{HgTe} / \mathrm{Ag}_{2} \\
\mathrm{Te} / \mathrm{HgTe} / \mathrm{Au}\end{array}$ & PV & 400 & $\begin{array}{c}4 \\
2.5\end{array}$ & $\begin{array}{l}85 \\
85\end{array}$ & 0.4 & $\begin{array}{l}3 \times 10^{11} \\
3 \times 10^{11}\end{array}$ & $2.5 \mu \mathrm{s}$ & $2019 / 45$ \\
\hline $\begin{array}{l}\text { FTO/HgTe4k- } \\
\text { ink/HgTe:6k/Au }\end{array}$ & PV & 560 & 2.5 & 300 & 0.003 & $3 \times 10^{9}$ & $\begin{array}{c}260 \\
\mathrm{~ns}\end{array}$ & $2019 / 42$ \\
\hline $\mathrm{Al}_{2} \mathrm{O}_{3} / \mathrm{TO} / \mathrm{HgTe}: \mathrm{HgCl}_{2} / \mathrm{Au}$ & PV & 400 & 2.5 & 300 & 1 & $5 \times 10^{10}$ & $1.4 \mu \mathrm{s}$ & $2020 / 40$ \\
\hline $\mathrm{Au} / \mathrm{HgTe}$ & PC & & $\begin{array}{c}5 \\
3.5\end{array}$ & $\begin{array}{l}300 \\
300\end{array}$ & $\begin{array}{l}0.015 \\
0.22\end{array}$ & $\begin{array}{l}5.4 \times 10^{9} \\
3.5 \times 10^{8}\end{array}$ & $\begin{array}{l}2.2 \\
\mathrm{~ms}\end{array}$ & $2020 / 46$ \\
\hline $\mathrm{SiO}_{2} / \mathrm{P} 3 \mathrm{HT}: \mathrm{HgTe} / \mathrm{Au}$ & PT & & 2.5 & 300 & 1 & $10^{10}$ & $1.5 \mu \mathrm{s}$ & $2020 / 47$ \\
\hline $\mathrm{Au} / \mathrm{LaF}_{3} / \mathrm{HgTe} / \mathrm{Graphene}$ & PT & 200 & 2.6 & 220 & 0.006 & $10^{9}$ & $7 \mu \mathrm{s}$ & $2020 / 48$ \\
\hline $\mathrm{Au} / \mathrm{HgTe} / \mathrm{Au}$ (nanogap) & PC & 300 & 2.5 & 200 & 1000 & $2 \times 10^{12}$ & $25 \mu \mathrm{s}$ & $2020 / 49$ \\
\hline $\begin{array}{l}\text { FTO/Al-GMR/HgTe- } \\
\text { ink/Ag2Te/Au }\end{array}$ & PV & 135 & 2 & 300 & 0.2 & $2 \times 10^{10}$ & $\begin{array}{l}110 \\
\mathrm{~ns}\end{array}$ & $\begin{array}{l}\text { This } \\
\text { work }\end{array}$ \\
\hline
\end{tabular}

\section{Conclusion}

In conclusion, we have determined the complex optical indices of $\mathrm{HgTe} \mathrm{NC}$ thin films using ellipsometry spectroscopy. Conveniently, electromagnetic simulations give satisfactory results when using a constant value for the refractive index equal to its spectral average. This typical mean value is $n \approx 2.35 \pm 0.15$, and is less driven by the band-gap than the surface chemistry/film deposition method. We then designed a diode that incorporates a resonator with a thin absorbing layer. We showed that a careful design of the resonator can be used to achieve broadband enhanced absorption ( $\approx 1000 \mathrm{~nm}$ large with a mean value above $75 \%$ ) from an absorbing film thickness below $150 \mathrm{~nm}$. The fabrication of the grating from aluminum also increases the work-function difference between the two electrodes resulting in further enhancement of the diode open-circuit voltage. The

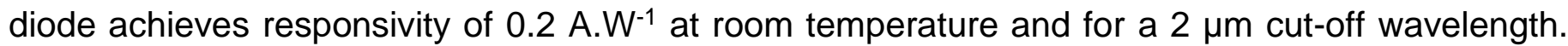
The specific detectivity is $2 \times 10^{10}$ Jones, while the time response is as short as $110 \mathrm{~ns}$. 


\section{METHODS}

Chemicals: Mercury chloride $\left(\mathrm{HgCl}_{2}\right.$, Sigma-Aldrich, $\left.99 \%\right)$, mercury compounds are highly toxic. Handle them with special care. tellurium powder (Te, Sigma-Aldrich, 99.99\%), silver nitrate $\left(\mathrm{AgNO}_{3}\right.$, Prolabo, 99.5\%), trioctylphosphine (TOP, Alfa, $90 \%$ ), oleic acid (OA, Sigma, 90\%), oleylamine (OLA, Acros, 80-90\%), dodecanethiol (DDT, Sigma-Aldrich, 98\%), trioctylamine (TOA, Aldrich), chloroform (Carlo Erba), 1,2 ethanedithiol (EDT, Fluka, 98\%), ethanol absolute anhydrous (VWR), methanol (VWR, >98\%), isopropanol (IPA, VWR), hexane (VWR, 99\%), octane (Carlo erba, 99\%), acetonitrile (VWR, 99.9\%), 2, mercaptoethanol (MPOH, Merck, >99\%), N,N dimethylformamide (DMF, VWR), chlorobenzene (VWR), toluene (VWR, $99.8 \%$ ). All chemicals are used without further purification.

$1 \mathrm{M}$ TOP:Te precursor: $2.54 \mathrm{~g}$ of Te powder is mixed in $20 \mathrm{~mL}$ of TOP in a three neck-flask. The flask is kept under vacuum at room temperature for $5 \mathrm{~min}$ and then the temperature is raised to 100 ${ }^{\circ} \mathrm{C}$. Furthermore, degassing of flask is conducted for the next $20 \mathrm{~min}$. The atmosphere is switched to nitrogen and the temperature is raised to $275^{\circ} \mathrm{C}$. The solution is stirred until a clear orange coloration is obtained. The flask is cooled down to room temperature and the color switches to yellow. Finally, this solution is transferred to a nitrogen filled glove box for storage.

HgTe NCs synthesis with band-edge at $6000 \mathrm{~cm}^{-1}(1.5 \mu \mathrm{m})$ : In a $100 \mathrm{~mL}$ three-neck flask, 540 $\mathrm{mg}$ of $\mathrm{HgCl}_{2}$ and $50 \mathrm{~mL}$ of oleylamine are degassed under vacuum at $110^{\circ} \mathrm{C}$. At this stage, the solution is yellow and clear. Meanwhile $2 \mathrm{~mL}$ of TOP:Te $(1 \mathrm{M})$ is extracted from the glove box and mixed with $8 \mathrm{~mL}$ of OLA. The atmosphere is switched to nitrogen and the temperature is set at $57^{\circ} \mathrm{C}$. The pre-heated TOP:Te solution is quickly injected and the solution turned dark after 1 min. After $3 \mathrm{~min}, 10 \mathrm{~mL}$ of a mixture of $20 \%$ DDT in toluene is injected and a water bath is used to quickly decrease the temperature. The content of the flask is separated in 4 tubes and methanol is added. After centrifugation, the formed pellets are redispersed in one falcon with $10 \mathrm{~mL}$ of toluene. The solution is precipitated a second time with absolute ethanol. Again, the formed pellets are redispersed in $8 \mathrm{~mL}$ of toluene. At this step the nanocrystals are centrifuged in pure toluene to get rid of the lamellar phase. The solid phase is discarded and the supernatant filtrated. The procedure for other sizes of $\mathrm{HgTe}$ NCs are given in the Sl.

\section{Film preparation}

HgTe ink: $1 \mathrm{~mL}$ of HgTe NC solution in toluene is mixed with $1 \mathrm{~mL}$ of exchange solution (15 mg of $\mathrm{HgCl}_{2}$ in $9 \mathrm{~mL}$ DMF and $1 \mathrm{~mL}$ of 2, mercaptoethanol). $5 \mathrm{~mL}$ of hexane is added to the solution and mixed through vortex and sonication. After few seconds two phases are clearly visible and the top one is removed. The solution is washed with hexane one more time. At this step $100 \mu \mathrm{L}$ of DMF is added to avoid a precipitation of the NCs. The solution is cleaned again with hexane. Then, the solution is precipitated by adding toluene and centrifuged at $6000 \mathrm{rpm}$ for $4 \mathrm{~min}$. The supernatant is discarded and the NCs are redispersed in $100 \mu \mathrm{L}$ of DMF.

HgTe solid state ligand exchange toward ethanedithiol: $50 \mathrm{mg} \cdot \mathrm{mL}^{-1} \mathrm{HgTe}$ in toluene is spin-coated at $2000 \mathrm{rpm}$ during $60 \mathrm{~s}$ on clean glass substrate. A solid-state ligand exchange is performed by dipping the substrate in $1 \%$ EDT $(\mathrm{v} / \mathrm{v})$ solution in ethanol for $30 \mathrm{~s}$. After that, the substrate is rinsed in pure ethanol. This procedure is repeated until the desired thickness is achieved. 


\section{MATERIAL CHARACTERIZATION}

Electron microscopy: For transmission electron microscopy (TEM) pictures, a drop of the NCs solution is drop-casted onto a copper grid covered with an amorphous carbon film. The grid is degassed overnight to reduce future contamination. A JEOL 2010F is used for acquisition of pictures and operated at $200 \mathrm{kV}$.

Optical ellipsometry: The spectroscopic ellipsometry measures the changes in the polarization state between the incident and the reflected light and is characterized by the angles $\psi$ and $\Delta$.

$$
\rho=\frac{r_{p}}{r_{s}}=\left|\frac{r_{p}}{r_{s}}\right| e^{i\left(\delta_{p}-\delta_{s}\right)}=\tan \psi e^{i \Delta}
$$

where $r_{p}$ and $r_{s}$ are the reflection coefficients of $p$ and s polarized light respectively and where $\delta_{p}$.and $\delta_{p}$ are the phase shifts in reflection in $\mathrm{p}$ and s polarizations, respectively. The measurements are performed on a V-VASE ellipsometer (J.A. Woollam) in the 350-2500 nm range with steps of $10 \mathrm{~nm}$ and with angles of incidence of $55^{\circ}$ and $65^{\circ}$.

Infrared spectroscopy: The solution of nanocrystal is drop-casted onto cleaned glass substrate for the sample in the near-infrared. For sample with the reddest band edge ( $\mathrm{HgTe} 2 \mathrm{k}$ corresponding to a $5 \mu \mathrm{m}$ cut-off wavelength), the glass absorbs at the wavelength corresponding to the band-edge, thus the nanocrystal film is deposited on sapphire substrate. The sample is then introduced into the Fourier Transform Infrared spectrometer (FTIR, Thermo Fischer iS50). Two sources have been used: a white light in the near infrared and a globar in the mid infrared. Two beam splitters have been used: $\mathrm{a} \mathrm{CaF}_{2}$ beam splitter in the near infrared and an extended $\mathrm{KBr}$ in the mid infrared. Two detectors have been used: an InGaAs sensor in the near infrared and a DTGS in the mid infrared. In all cases, the background uses the same optical configuration but without sample onto the substrate. Spectra are typically acquired with a $4 \mathrm{~cm}^{-1}$ resolution and typically averaged 32 times.

Reflectivity measurements are conducted using a Brucker Vertex 70v Fourier transform Infrared spectrometer. To measure the diode reflection, we use the spectrometer in reflection configuration under ambient atmosphere and at room temperature. The source is a tungsten lamp and the detector is a DLaTGS. A CaF 2 beam-splitter is used. Spectra are typically acquired with a $4 \mathrm{~cm}^{-1}$ resolution and averaging over 32 spectra at least. The reference is made with a gold mirror.

Electromagnetic simulation: Calculations are achieved with Matlab library based on RCWA ${ }^{50}$. The Maxwell equations are solved in each layer and interface conditions are applied to find the final solution of the whole structure. We consider incoming plane waves under normal incidence, either with transverse magnetic (TM) or transverse electric (TE) polarizations, i.e. with magnetic field or electric field parallel with the slits of the gratings, respectively. In 1D simulations, the grating is supposed to be invariant along $y$ direction, and repeated infinitely along $x$ direction. In 2D simulations the grating is repeated infinitely along $x$ and $y$ directions. The inputs needed for this simulation are the device structure and the optical index of the different materials.

Device Fabrication: For the device fabrication $70 \mathrm{~nm}$ FTO $\left(\rho_{\mathrm{s}} \approx 70-90 \Omega / \mathrm{sq}\right)$ on glass substrate from Solems are used. To define the device area, FTO electrodes are patterned with optical UV lithography as described in ref. ${ }^{42}$ Before using it, the FTO/glass substrate is cleaned with acetone 
and IPA. The HgTe $1.7 \mu$ NCs ink is deposited onto the patterned FTO substrate via spin-coating. The thickness of the film is tuned with spin-coating speed and ink concentration in DMF solvent. On top of $\mathrm{HgTe}$ ink layer $\mathrm{Ag}_{2} \mathrm{Te}$ nanocrystals layer is spin-coated at $2000 \mathrm{rpm}$ followed by $\mathrm{HgCl}_{2}$ treatment. For $\mathrm{HgCl}_{2}$ treatment, $50 \mu \mathrm{L}$ of $\mathrm{HgCl}_{2}$ methanol $(10 \mathrm{mM})$ solution is dropped onto $\mathrm{HgTe}$ film and spin-dried after $15 \mathrm{~s}$. Then the film is rinsed with IPA. This procedure is repeated twice. Finally, an EDT ligand exchange is performed by dipping the film in 1\% EDT acetonitrile solution for $30 \mathrm{~s}$ and rinsed with pure acetonitrile. A $80 \mathrm{~nm}$ Au top electrode is deposited with thermal evaporation under a vacuum of $\approx 10^{-6} \mathrm{mbar}$ at the rate of $3 \AA / \mathrm{s}$. The thickness is monitored with an in situ quartz crystal. The substrate holder is rotated throughout the deposition to ensure an homogeneous thickness. The overlap area of both electrodes FTO and Au is defined as the device area of $1 \mathrm{~mm}^{2}$.

Device Characterization: The devices are operated in room conditions of pressure and temperature. The sample is connected to a Keithley 2634 used as a source-meter that applied bias and measure current. For measurements under illumination, we use two types of source: a blackbody (omega BB4B) typically operated at $600^{\circ} \mathrm{C}$ and $980{ }^{\circ} \mathrm{C}$ used as a broad band source and a $1.55 \mu \mathrm{m}$ laser diode particularly used for time response.

Time-resolved measurements: For the time-resolved measurements, we use a pulsed laser (Crylas FTSS 355-50) at $355 \mathrm{~nm}$. The laser beam is around $1 \mathrm{~mm}$ in diameter (i.e. poorly focused) to prevent excessive heating of the sample, which may lead to a drift of the photoresponse. The pulses are $1 \mathrm{~ns}$ long and repeated every $10 \mathrm{~ms}(100 \mathrm{~Hz})$. A photodiode is used to trigger the signal. An oscilloscope (Rohde \& Schwarz, RTE 1102) acquires the outcoming signal through a $50 \Omega$ resistor, which is proportional to the current. 


\section{Supporting Information}

Supporting Information is available from the Wiley Online Library.

It includes information relative nonocrystals synthesis, ellipsometry data and process for diode fabrication. Spectrally resolved Data for complex optical index of $\mathrm{HgT} \mathrm{NC}$ thin film are available in the supplementary material of this article.

\section{ACKNOWLEDEGMENTS}

The project is supported by ERC starting grant blackQD (grant $n^{\circ} 756225$ ). S.I. thanks the support ERC starting grant Ne2Dem (grant $n^{\circ} 853,049$ ). We acknowledge the use of clean-room facilities from the "Centrale de Proximité Paris-Centre". This work has been supported by the Region lle-deFrance in the framework of DIM Nano-K (grant dopQD). This work was supported by French state funds managed by the ANR within the Investissements d'Avenir programme under reference ANR11-IDEX-0004-02, and more specifically within the framework of the Cluster of Excellence MATISSE and also by the grant IPER-Nano2, Copin (ANR-19-CE24-0022), Frontal (ANR-19-CE09-0017), Graskop (ANR-19-CE09-0026) and NITQuantum. JQ thanks Chinese Scholarship Council for PhD funding while $A C$ thanks Agence innovation defense.

\section{Conflict of Interest}

The authors declare no conflict of interest.

Keywords: narrow band gap nanocrystals, $\mathrm{HgTe}$, short and mid wave infrared, optical index, ellipsometry, plasmonic resonator, cavity.

\section{REFERENCES}

(1) Hafiz, S. Bin; Scimeca, M.; Sahu, A.; Ko, D. K. Colloidal quantum dots for thermal infrared sensing and imaging. Nano Converg. 2019, 6, 7.

(2) Lhuillier, E.; Guyot-Sionnest, P. Recent Progresses in Mid Infrared Nanocrystal Optoelectronics. IEEE J. Sel. Top. Quantum Electron. 2017, 23, 6000208.

(3) Georgitzikis, E.; Malinowski, P. E.; Li, Y.; Maes, J.; Hagelsieb, L. M.; Guerrieri, S.; Hens, Z.; Heremans, P.; Cheyns, D. Integration of PbS Quantum Dot Photodiodes on Silicon for NIR Imaging. IEEE Sens. J. 2019, 6841-6848.

(4) Rauch, T.; Böberl, M.; Tedde, S. F.; Fürst, J.; Kovalenko, M. V; Hesser, G.; Lemmer, U.; Heiss, W.; Hayden, O. Near-infrared imaging with quantum-dot-sensitized organic photodiodes. Nat. Photonics 2009, 3, 332-336.

(5) Chu, A.; Martinez, B.; Ferré, S.; Noguier, V.; Gréboval, C.; Livache, C.; Qu, J.; Prado, Y.; Casaretto, N.; Goubet, N.; Cruguel, H.; Dudy, L.; Silly, M. G.; Vincent, G.; Lhuillier, E. HgTe Nanocrystals for SWIR Detection and Their Integration up to the Focal Plane Array. ACS Appl. Mater. Interfaces 2019, 11, 33116-33123.

(6) Gréboval, C.; Ferre, S.; Noguier, V.; Chu, A.; Qu, J.; Chee, S.-S.; Vincent, G.; Lhuillier, E. narrow band gap nanocrystals: recent progresses relative to imaging and active detectiolnfraredn. arXiv:2001.11554 2020.

(7) Buurma, C.; Pimpinella, R. E.; Ciani, A. J.; Feldman, J. S.; Grein, C. H.; Guyot-Sionnest, P. MWIR imaging with low cost colloidal quantum dot films. SPIE 2016, 9933, 993303.

(8) Ciani, A. J.; Pimpinella, R. E.; Grein, C. H.; Guyot-Sionnest, P. Colloidal quantum dots for lowcost MWIR imaging. SPIE 2016, 9819, 333-341.

(9) Malinowski, P. E.; Georgitzikis, E.; Maes, J.; Vamvaka, I.; Frazzica, F.; Van Olmen, J.; De Moor, P.; Heremans, P.; Hens, Z.; Cheyns, D. Thin-film quantum dot photodiode for monolithic infrared image sensors. Sensors 2017, 17, 2867.

(10) Hinds, S.; Klem, E.; Gregory, C.; Hilton, A.; Hames, G.; Violette, K. Extended SWIR high performance and high definition colloidal quantum dot imagers. Infrared Technol. Appl. XLVI 
2020, 11407, 1140707.

(11) Georgitzikis, E.; Malinowski, P. E.; Hagclsieb, L. M.; Pejovic, V.; Uytterhoeven, G.; Guerrieri, S.; Süss, A.; Cavaco, C.; Chatzinis, K.; Maes, J.; Hens, Z.; Heremans, P.; Cheyns, D. NIR Sensors Based on Photolithographically Patterned PbS QD Photodiodes for CMOS Integration. IEEE SENSORS 2018, 18329605.

(12) Georgitzikis, E.; Malinowski, P.; Maes, J.; Gielen, S.; Frazzica, F.; Li, Y.; Boulenc, P.; Lee, J.; Cavaco, C.; Guerrieri, S.; Hens, Z.; Heremans, P.; Cheyns, D. Thin-film photodetectors for NIR and SWIR Image Sensors with $0.13 \mu \mathrm{m}$ tech node CMOS read-out. Freibg. Infrared Colloq. 44th 2019.

(13) Bakulin, A.; D’Souza, A. I.; Masterjohn, C.; Mei, E.; Li, C.; Klem, E.; Temple, D. ROIC for 3 $\mu \mathrm{m}$ Pixel Pitch Colloidal Quantum Dot Detectors. Image Sens. Technol. Mater. Devices, Syst. Appl. V 2018, 10656, 164-174.

(14) Livache, C.; Izquierdo, E.; Martinez, B.; Dufour, M.; Pierucci, D.; Keuleyan, S.; Cruguel, H.; Becerra, L.; Louis Fave, J.; Aubin, H.; Ouerghi, A.; Lacaze, E.; G. Silly, M.; Dubertret, B.; Ithurria, S.; Lhuillier, E. Charge Dynamics and Optolectronic Properties in HgTe Colloidal Quantum Wells. Nano Lett. 2017, 17, 4067-4074.

(15) Izquierdo, E.; Robin, A.; Keuleyan, S.; Lequeux, N.; Lhuillier, E.; Ithurria, S. Strongly Confined HgTe 2D Nanoplatelets as Narrow Near-Infrared Emitters. J. Am. Chem. Soc. 2016, 138, 10496-10501.

(16) Moghaddam, N.; Greboval, C.; Qu, J.; Chu, A.; Rastogi, P.; Livache, C.; Khalili, A.; Xu, X. Z.; Baptiste, B.; Klotz, S.; Fishman, G.; Capitani, F.; Ithurria, S.; Sauvage, S.; Lhuillier, E. The Strong Confinement Regime in HgTe Two-Dimensional Nanoplatelets. J. Phys. Chem. C 2020, 124, 23460-23468.

(17) Lhuillier, E.; Scarafagio, M.; Hease, P.; Nadal, B.; Aubin, H.; Zhen Xu, X.; Lequeux, N.; Patriarche, G.; Ithurria, S.; Dubertret, B. Infrared Photodetection Based on Colloidal QuantumDot Films with High Mobility and Optical Absorption up to THz. Nano Lett. 2016, 16, 12821286.

(18) Goubet, N.; Jagtap, A.; Livache, C.; Martinez, B.; Portalès, H.; Xu, X. Z.; Lobo, R. P. S. M.; Dubertret, B.; Lhuillier, E. Terahertz HgTe Nanocrystals: Beyond Confinement. J. Am. Chem. Soc. 2018, 140, 5033-5036.

(19) Jagtap, A.; Martinez, B.; Goubet, N.; Chu, A.; Livache, C.; Gréboval, C.; Ramade, J.; Amelot, D.; Trousset, P.; Triboulin, A.; Ithurria, S.; Silly, M. G.; Dubertret, B.; Lhuillier, E. Design of a Unipolar Barrier for a Nanocrystal-Based Short-Wave Infrared Photodiode. ACS Photonics 2018, 5, 4569-4576.

(20) Tang, X.; M. Ackerman, M.; Guyot-Sionnest, P. Thermal Imaging with Plasmon Resonance Enhanced HgTe Colloidal Quantum Dot Photovoltaic Devices. ACS Nano 2018, 12, 73627370.

(21) Tang, X.; Ackerman, M. M.; Guyot-Sionnest, P. Acquisition of Hyperspectral Data with Colloidal Quantum Dots. Laser Photon. Rev. 2019, 13, 1900165.

(22) Gréboval, C.; Chu, A.; Magalhaes, D. V.; Ramade, J.; Junling Qu; Rastogi, P.; Khalili, A.; Chee, S.-S.; Aubin, H.; Vincent, G.; Bals, S.; Delerue, C.; Lhuillier, E. Ferroelectric Gating of Narrow Band-Gap Nanocrystal Arrays with Enhanced Light Matter Coupling. ACS Photonics 2020, Submitted.

(23) Tang, X.; Wu, G. fu; Lai, K. W. C. Plasmon resonance enhanced colloidal HgSe quantum dot filterless narrowband photodetectors for mid-wave infrared. J. Mater. Chem. C 2017, 5, 362369.

(24) Chu, A.; Gréboval, C.; Goubet, N.; Martinez, B.; Livache, C.; Qu, J.; Rastogi, P.; Bresciani, F. A.; Prado, Y.; Suffit, S.; Ithurria, S.; Vincent, G.; Lhuillier, E. Near Unity Absorption in Nanocrystal Based Short Wave Infrared Photodetectors Using Guided Mode Resonators. ACS Photonics 2019, 6, 2553-2561.

(25) Sergeev, A. A.; Pavlov, D. V; Kuchmizhak, A. A.; Lapine, M. V; Yiu, W. K.; Dong, Y.; Ke, N.; Juodkazis, S.; Zhao, N.; Kershaw, S. V; Rogach, A. L. Tailoring spontaneous infrared emission of HgTe quantum dots with laser-printed plasmonic arrays. Light Sci. Appl. 2020, 9, 16.

(26) Geiregat, P.; Houtepen, A. J.; Sagar, L. K.; Infante, I.; Zapata, F.; Grigel, V.; Allan, G.; Delerue, 
C.; Van Thourhout, D.; Hens, Z. Continuous-wave infrared optical gain and amplified spontaneous emission at ultralow threshold by colloidal $\mathrm{HgTe}$ quantum dots. Nat. Mater. 2018, $17,35-42$.

(27) Diroll, B. T.; Gaulding, E. A.; Kagan, C. R.; Murray, C. B. Spectrally-Resolved Dielectric Functions of Solution-Cast Quantum Dot Thin Films. Chem. Mater. 2015, 27, 6463-6469.

(28) Lhuillier, E.; Keuleyan, S.; Guyot-Sionnest, P. Optical properties of HgTe colloidal quantum dots. Nanotechnology 2014, 25, 189501.

(29) Szuszkiewicz, W.; Witowski, A. M.; Grynberg, M. The Dynamic Dielectric Function in HgSe and HgTe. Phys. Status Solidi 1978, 87, 637-645.

(30) Secuk, M. N.; Aycibin, M.; Erdinc, B.; Gulebaglan, S. E.; Dogan, E. K.; Akkus, H. Ab-initio Calculations of Structural , Electronic, Optical , Dynamic and Thermodynamic Properties of HgTe and HgSe. Am. J. Condens. Matter Phys. 2014, 4, 13-19.

(31) El-Nahass, M. M.; El-Salam, F. A.; Seyam, M. A. M. Optical and structural properties of flash evaporated HgTe thin films. J. Mater. Sci. 2006, 41, 3573-3580.

(32) Moritani, A.; Sekiya, H.; Taniguchi, K.; Hamaguchi, C.; Nakai, J.; Makabe, R. Optical constants of HgTe and hgse. Jpn. J. Appl. Phys. 1971, 10, 1410-1414.

(33) Bejaoui, A.; Alonso, M. I.; Garriga, M.; Campoy-Quiles, M.; Goñi, A. R.; Hetsch, F.; Kershaw, S. V; Rogach, A. L.; To, C. H.; Foo, Y.; Zapien, J. A. Evaluation of the dielectric function of colloidal $\mathrm{Cd}_{1-\mathrm{x}} \mathrm{Hg}_{\mathrm{x}} \mathrm{Te}$ quantum dot films by spectroscopic ellipsometry. Appl. Surf. Sci. 2017, 421, 295-300.

(34) Laref, A.; Alsagri, M.; Laref, S.; Yang, J. T.; Xiong, Y. C.; Khandy, S. A. Relativistic effects on the electronic and optical characteristics of $\mathrm{Cd1}$-xHgxTe alloys-based solar cell materials. J. Phys. Chem. Solids 2019, 129, 368-377.

(35) Losurdo, M.; Bergmair, M.; Bruno, G.; Cattelan, D.; Cobet, C.; de Martino, A.; Fleischer, K.; Dohcevic-Mitrovic, Z.; Esser, N.; Galliet, M.; Gajic, R.; Hemzal, D.; Hingerl, K.; Humlicek, J.; Ossikovski, R.; Popovic, Z. V; Saxl, O. Spectroscopic ellipsometry and polarimetry for materials and systems analysis at the nanometer scale: state-of-the-art, potential, and perspectives. J. Nanoparticle Res. 2009, 11, 1521-1554.

(36) Adachi, S. Handbook on Physical Properties of Semiconductors; Kluwer Academic Publishers: Springer, Boston, 2004.

(37) Keuleyan, S.; Lhuillier, E.; Guyot-Sionnest, P. Synthesis of colloidal HgTe quantum dots for narrow mid-IR emission and detection. J. Am. Chem. Soc. 2011, 133, 16422-16424.

(38) Ning, Y.; Zhang, S.; Hu, Y.; Hao, Q.; Tang, X. Simulation of Monolithically Integrated MetaLens with Colloidal Quantum Dot Infrared Detectors for Enhanced Absorption. Coatings 2020, $10,1218$.

(39) Hafiz, S. Bin; Al Mahfuz, M. M.; Ko, D. K. Vertically stacked intraband quantum dot devices for mid-wavelength infrared photodetection. ACS Appl. Mater. Interfaces 2021, 13, 927-943.

(40) Ackerman, M. M.; Chen, M.; Guyot-Sionnest, P. HgTe colloidal quantum dot photodiodes for extended short-wave infrared detection. Appl. Phys. Lett. 2020, 116, 083502.

(41) Ackerman, M. M.; Tang, X.; Guyot-Sionnest, P. Fast and Sensitive Colloidal Quantum Dot Mid-Wave Infrared Photodetectors. ACS Nano 2018, 12, 7264-7271.

(42) Martinez, B.; Ramade, J.; Livache, C.; Goubet, N.; Chu, A.; Gréboval, C.; Qu, J.; Watkins, W. L.; Becerra, L.; Dandeu, E.; Fave, J. L.; Méthivier, C.; Lacaze, E.; Lhuillier, E. HgTe Nanocrystal Inks for Extended Short-Wave Infrared Detection. Adv. Opt. Mater. 2019, 7, 1900348.

(43) Chen, H.-L.; Cattoni, A.; De Lépinau, R.; Walker, A. W.; Höhn, O.; Lackner, D.; Siefer, G.; Faustini, M.; Vandamme, N.; Goffard, J.; Behaghel, B.; Dupuis, C.; Bardou, N.; Dimroth, F.; Collin, S. A 19.9\%-efficient ultrathin solar cell based on a 205-nm-thick GaAs absorber and a silver nanostructured back mirror. Nat. Energy 2019, 4, 761-767.

(44) Chen, M.; Lu, H.; Abdelazim, N. M.; Zhu, Y.; Wang, Z.; Ren, W.; Kershaw, S. V; Rogach, A. L.; Zhao, N. Mercury Telluride Quantum Dot Based Phototransistor Enabling High-Sensitivity Room-Temperature Photodetection at $2000 \mathrm{~nm}$. ACS Nano 2017, 11, 5614-5622.

(45) Tang, X.; Ackerman, M. M.; Chen, M.; Guyot-Sionnest, P. Dual-band infrared imaging using stacked colloidal quantum dot photodiodes. Nat. Photonics 2019, 13, 277-282.

(46) Cryer, M. E.; Browning, L. A.; Plank, N. O. V.; Halpert, J. E. Large Photogain in Multicolor 
Nanocrystal Photodetector Arrays Enabling Room-Temperature Detection of Targets Above $100^{\circ} \mathrm{C}$. ACS Photonics 2020, 7, 3078-3085..

(47) Dong, Y.; Chen, M.; Yiu, W. K.; Zhu, Q.; Zhou, G.; Kershaw, S. V.; Ke, N.; Wong, C. P.; Rogach, A. L.; Zhao, N. Solution Processed Hybrid Polymer: HgTe Quantum Dot Phototransistor with High Sensitivity and Fast Infrared Response up to $2400 \mathrm{~nm}$ at Room Temperature. Adv. Sci. 2020, 7, 2000068.

(48) Noumbé, U. N.; Gréboval, C.; Livache, C.; Chu, A.; Majjad, H.; Parra López, L. E.; Mouafo, L. D. N.; Doudin, B.; Berciaud, S.; Chaste, J.; Ouerghi, A.; Lhuillier, E.; Dayen, J. F. Reconfigurable 2D/0D p-n Graphene/HgTe Nanocrystal Heterostructure for Infrared Detection. ACS Nano 2020, 14, 4567-4576.

(49) Chu, A.; et al. Infrared Photoconduction at the Diffusion Length Limit in HgTe Nanocrystal Array. Submitted 2020.

(50) Hugonin, J. P.; Lalanne, P. Reticolo Software for Grating Analysis; 2005. 


\section{Table of content graphic}

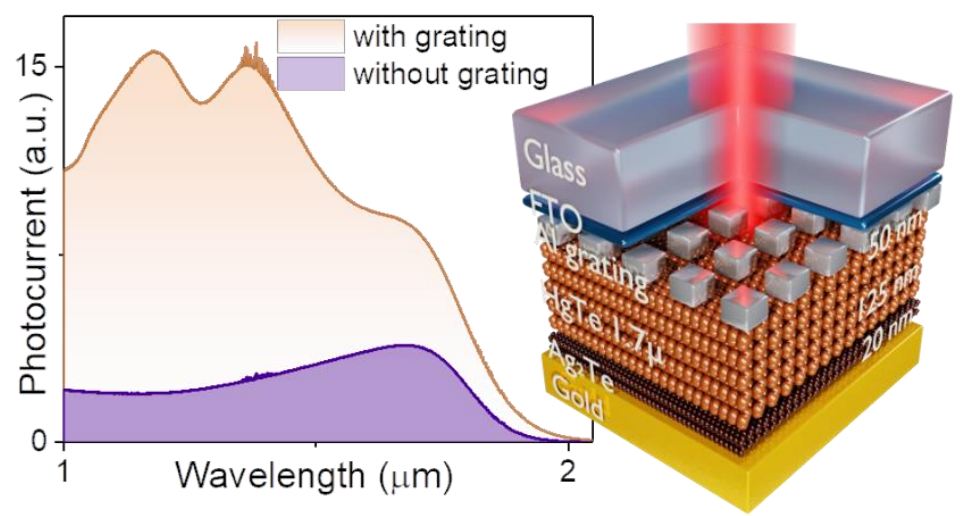

